\title{
Interaction of Ligands with Acetylcholinesterase. Use of Temperature- Jump Relaxation Kinetics in the Binding of Specific Fluorescent Ligands ${ }^{\dagger}$
}

\author{
Terrone L. Rosenberry* and Eberhard Neumann
}

ABSTRACT: The fluorescence of either $N$-methylacridinium (I) or 1-methyl-7-hydroxyquinolinium (II) is totally quenched on binding to the catalytic site of acetylcholinesterase. Equilibrium titrations of $11 \mathrm{~S}$ acetylcholinesterase at $0.1 \mathrm{M}$ ionic strength with I confirmed previous reports that binding shows high specificity for the catalytic site. Analogous titrations with II indicated that only the protonated, cationic form of II binds and that binding has a specificity and stoichiometry similar to that of $I$. Under most of the experimental conditions introduced here, the reaction of either I or II with acetylcholinesterase was characterized by a single relaxation time. The bimolecular association constants for the reaction were unusually high, at $23^{\circ} \mathrm{C}$ and $\sim 0.1 \mathrm{M}$ ionic strength; for $\mathrm{I}, k_{12}=1.18 \pm$ $0.03 \times 10^{9} \mathrm{M}^{-1} \mathrm{~s}^{-1}$; for II, $k_{12}=2.18 \pm 0.15 \times 10^{9} \mathrm{M}^{-1} \mathrm{~s}^{-1}$. These constants were obtained from observed relaxation times both by a conventional analysis of equilibrium reactant con-

$T_{1}$ he speed with which acetylcholinesterase catalyzes the hydrolysis of acetylcholine has long been appreciated. Studies by Michel and Krop (1951), Lawler (1961), Kremzner and Wilson (1964) and others are in quite close agreement on a maximum turnover number $\left(k_{\text {cat }}\right)$ of $1.6 \times 10^{4} \mathrm{~s}^{-1}$ at $\mathrm{pH} 8$ and $25^{\circ} \mathrm{C}$ (Rosenberry and Bernhard, 1971; Rosenberry, 1975a). Recently it has been emphasized (Rosenberry, 1975a) that this enzymatic hydrolysis is also extremely efficient at the perhaps more physiologically relevant acetylcholine concentrations below the apparent Michaelis constant $K_{\text {app }}(<0.1 \mathrm{mM})$. At these concentrations the appropriate rate parameter is the second-order rate constant $k_{\text {cat }} / K_{\text {app. }}$ Values of $k_{\text {cat }} / K_{\text {app }}$ for acetylcholine and several other cationic substrates are around $2 \times 10^{8} \mathrm{M}^{-1} \mathrm{~s}^{-1}$ at $25^{\circ} \mathrm{C}$ and $0.1 \mathrm{M}$ ionic strength (Rosenberry, 1975a) and thus approach a limit generally expected for diffusion-controlled enzyme reactions (Eigen and Hammes, 1963). In agreement with this suggestion, a low deuterium oxide isotope effect of 1.1 is associated with $k_{\text {cat }} / K_{\text {app }}$ for acetylcholine (Rosenberry, 1975b); this observation indicates that the rate-limiting step for acetylcholine hydrolysis at low substrate concentrations precedes general acid-base catalysis and is likely to involve either the bimolecular reaction step or a subsequent conformational change of the enzyme-substrate complex (Rosenberry, 1975b).

Because of the importance of the initial steps associated with the interaction of acetylcholinesterase with specific ligands, we have used temperature-jump relaxation kinetics to investigate the reactions of this enzyme with $N$-methylacridinium

\footnotetext{
+ From the Departments of Neurology and Biochemistry, College of Physicians and Surgeons, Columbia University, New York, New York 10032 and the Max-Planck-Institut fuer Biochemie, D-8033 Martinsried bei Muenchen, West Germany. Received December 14, 1976. This investigation was supported, in part, by United States Public Health Service Grant NS-03304, National Science Foundation Grant PCM73-00744. and a NATO Senior Fellowship in Science to T.L.R.
}

centrations and by a new method introduced here in which only the total ligand concentration need be known. At relatively high concentrations of enzyme and I, a second relaxation was observed; analyses of relaxation amplitudes indicated that this relaxation reflected independent ligand binding at a second, peripheral site on the enzyme. It has recently been suggested by $\mathrm{M}$. Eigen that certain specific ligands may have unusually high bimolecular association constants with their target macromolecules because they can bind initially to peripheral sites and proceed to the specific site by surface diffusion on the macromolecule. A test of this proposal for acetylcholinesterase and I was conducted by introducing $30 \mathrm{mM} \mathrm{Ca}^{2+}$ to the solvent. No supporting evidence was obtained. Nevertheless, this proposal, applied to other sites with very low ligand affinities, may still partially account for the high bimolecular association rate constants.

(I) and 1-methyl-7-hydroxyquinolinium (II). Both I and the zwitterionic form of II are highly fluorescent, and the fluorescence of both I and II is totally quenched when they bind<smiles>[H][Y]1c2ccccc2cc2ccccc12</smiles>

I

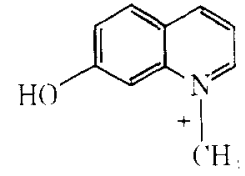

II with high affinity to acetylcholinesterase. Careful studies by Mooser et al. (1972), Mooser and Sigman (1974), and Taylor and Lappi (1975) have established that I binds with high specificity to each of the four independent catalytic sites in the 11S enzyme tetramer. The binding of II is less well defined, but kinetic data (Rosenberry and Bernhard, 1972) and our current study suggest that it is also highly specific for the catalytic sites.

In this study we show that these ligands bind to the enzyme with unusually high bimolecular rate constants. The possibility that peripheral anionic sites are contributing to these rate constants is considered

\section{Experimental Section}

Materials. Acetylcholinesterase from electric organs of the eel Electrophorus electricus was purified as an $11 \mathrm{~S}$ species free of detectable protein contaminants as described previously (Rosenberry et al., 1974). The preparation used in this study had been stored as a concentrated frozen solution for several months and had a specific activity of $5.7 \mathrm{mmol}$ of acetylcholine hydrolyzed $\mathrm{min}^{-1}$ ( $\mathrm{mg}$ of protein) $)^{-1}$ with $2.0 \mathrm{mM}$ acetylcholine in $0.1 \mathrm{M} \mathrm{NaCl}$ at $\mathrm{pH} 7.4$ and $25^{\circ} \mathrm{C}$. This corresponds to about $70 \%$ of the maximal specific activity observed for $11 \mathrm{~S}$ acetylcholinesterase. Normalities of acetylcholinesterase solutions were measured according to either eq 1 or eq 11-13 below. 
The recrystallized perchlorate salt of $N$-methylacridinium was a gift from Dr. Meir Shinitzky. A $\lambda_{\max }$ at $358 \mathrm{~nm}$ with $\epsilon_{358}$ $21300 \mathrm{M}^{-1} \mathrm{~cm}^{-1}$ was observed for this compound in $0.1 \mathrm{M}$ sodium phosphate buffer at $\mathrm{pH}$ 8.0.1-Methyl-7-hydroxyquinolinium iodide $\left(\lambda_{\max } 406 \mathrm{~nm}, \epsilon_{406} 9200 \mathrm{M}^{-1} \mathrm{~cm}^{-1}\right.$ in $0.1 \mathrm{M}$ sodium phosphate, $\mathrm{pH} 7.0$ ) was obtained by hydrolysis of 1methyl-7-acetoxyquinolinium iodide (Rosenberry and Bernhard, 1972). Sodium phosphate buffers labeled $0.1 \mathrm{M}$ were prepared by mixing appropriate amounts of $0.1 \mathrm{M} \mathrm{NaH}_{2} \mathrm{PO}_{4}$ and $0.05 \mathrm{M} \mathrm{Na}_{2} \mathrm{HPO}_{4}$ to achieve the indicated $\mathrm{pH}$.

Fluorescence Measurements. Most of the equilibrium and all of the kinetic data reported here were obtained in a fluorescence temperature-jump apparatus described previously (Rigler et al., 1974; Department of Biochemical Kinetics, Max-Planck-Institute for Biophysical Chemistry, Goettingen, West Germany). Excitation light from a mercury source was passed through a monochrometer adjusted to the adsorption maximum of the fluorescent ligand and focused on the sample compartment containing $0.8 \mathrm{~mL}$ of solution. Emission light was passed through cutoff filters, which removed scattered excitation light, and collected by two independent photomultiplier units placed at $90^{\circ}$ angles to the excitation light. Equilibrium fluorescence values were read as photomultiplier voltages normalized by a reference photomultiplier voltage recorded directly from the light source.

Fluorescence relaxation spectra were induced by a $3.3{ }^{\circ} \mathrm{C}$ temperature increase triggered by a $20-\mathrm{kV}$ discharge from a $5 \times 10^{-8} \mathrm{~F}$ capacitor through the sample compartment. The initial temperature was $20.0^{\circ} \mathrm{C}$ and the final temperature was $23 .{ }^{\circ} \mathrm{C}$. Transient photomultiplier signals were electrically damped to optimize signal to noise. In the presence of high equilibrium fluorescence, a time-delay device allowed monitoring of only those fluorescence relaxations of interest. Relaxation spectra were displayed on a calibrated Tektronix storage oscilloscope and recorded on $35-\mathrm{mm}$ film. Relaxation times and amplitudes were obtained by superposition of the film image on an oscilloscope screen whose voltage input derived from a calibrated multiple exponential function generator developed by C. R. Rabl (Max-Planck-Institute for Biophysical Chemistry, Goettingen, Germany). Exposure of acetylcholinesterase to the described temperature-jump experimentation for 4 to $6 \mathrm{~h}$ gave no detectable loss of enzyme activity.

A few equilibrium fluorescence measurements were also made on a Perkin-Elmer MPF-3A spectrofluorometer.

Ligand-Interaction Schemes. Three alternative schemes were considered for the interaction of fluorescent ligands with acetylcholinesterase.

SCHEME I

$$
\mathrm{E}+\mathrm{L} \underset{k_{21}}{\stackrel{k_{12}}{\rightleftharpoons}} \mathrm{EL}
$$

In Scheme I, ligands bind only to the catalytic site of the enzyme in a simple, one-step process given by a forward rate constant $k_{12}$ and a backward rate constant $k_{21}$. The ratio $k_{21} / k_{12}$ defines the equilibrium dissociation constant $K_{1}$. Schemes II and II' extend Scheme I by including a second li-

SCHEME II

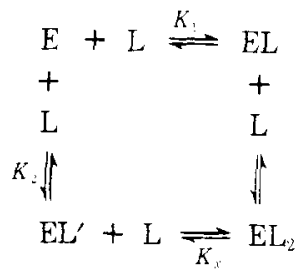

SCHEME II'

$$
\begin{aligned}
& \mathrm{E}+\mathrm{L} \underset{K_{l}}{\rightleftharpoons} \mathrm{EL} \\
& \mathrm{E}^{\prime}+\mathrm{L} \underset{k^{\prime}=1}{\stackrel{k_{1}^{\prime}}{\rightleftharpoons}} \mathrm{EL} L^{\prime}
\end{aligned}
$$

gand-binding site. In general, Scheme II could apply to any enzyme catalytic subunit which has two ligand-binding sites; however, when these two sites are assumed to bind ligand independently, $K_{1} \equiv K_{\mathrm{x}}$ and Scheme II becomes equivalent to Scheme $\mathrm{II}^{\prime}$. In Scheme II', E and $\mathrm{E}^{\prime}$ indicate independent binding-site species; interactions at the second site occur with rate constants $k_{12}{ }^{\prime}$ and $k_{21}{ }^{\prime}$ (equilibrium constant $k_{21}{ }^{\prime} / k_{12}{ }^{\prime}$ $\equiv K_{2}$ ).

\section{SCHEME III}

$$
\mathrm{E}+\mathrm{L} \stackrel{\kappa_{1}}{\rightleftharpoons} \mathrm{EL} \stackrel{k_{23}}{\underset{k_{32}}{\rightleftharpoons}} \mathrm{EL} *
$$

Scheme III assumes only a single ligand-binding site as in Scheme I. However, it extends Scheme I to include a second form of the enzyme-ligand complex EL* which is accessible via a conformational change of the initial complex EL. This conformational change has rate constants $k_{23}$ and $k_{32}$ and equilibrium constant $k_{32} / k_{23} \equiv K_{3}$.

Equilibrium Titrations. Our observations with $N$-methylacridinium are consistent with a previous report (Mooser et al., 1972) that the fluorescence of the ligand is totally quenched on binding to the enzyme (fluorescence of bound ligand $\leqslant 0.1 \%$ of fluorescence of free ligand). This is also the case for 1 methyl-7-hydroxyquinolinium. Consequently, the observed fluorescence $F$ is directly proportional to the free ligand concentration $c_{\mathrm{L}}$ such that $F=f_{C_{\mathrm{L}}}$, where $f$ is the fluorescence intensity coefficient obtained from observed linear $F$ vs. $\bar{c}_{1}$ plots in the absence of enzyme. For Scheme I, an equilibrium titration of enzyme with increasing amounts of ligand may be expressed by eq 1 ,

$$
\frac{1}{f B^{\prime}} \equiv \frac{1}{\left[1+\frac{\Delta V}{V}\right]\left[f \mathcal{C}_{\mathrm{L}}{ }^{\mathrm{ot}}-F\right]}=\frac{1}{f \mathcal{C}_{\mathrm{E}}^{\text {init }}}\left(1+\frac{f K_{1}}{F}\right)
$$

where $c_{L}{ }^{\text {tot }}$ is the total ligand concentration; $c_{E}$ init is the initial total enzyme concentration; $\Delta V / V$ is a dilution correction for incremental volume additions of ligand solution $\Delta V$ to initial total volume $V$; and $B^{\prime}$ is the concentration of bound ligand $B_{1}$. multiplied by a dilution correction factor. $F$ values were corrected for a slight background emission arising from solvent and enzyme alone. In a plot of $\left(f B^{\prime}\right)^{-1}$ vs. $F^{-1}, C_{E}^{\text {init }}$ is obtained from the $\left(f B^{\prime}\right)^{-1}$ axis intercept $\left(f_{\mathcal{C}} E^{\text {init }}\right)^{-1}$, and $K_{1}$ is obtained from the ratio $\left(f K_{1}\right)$ of the slope to the $\left(f B^{\prime}\right)^{-1}$ axis intercept. An equation similar to eq 1 was used by Mooser et al. (1972) in their equilibrium titration of acetylcholinesterase with $N$-methylacridinium. Given the observed total lack of bound ligand fluorescence, eq 1 would also hold for Scheme III if $K_{1}$ is replaced by the overall $K_{\text {III }}$, where $K_{1 I I}$ is given by eq 2

$$
K_{\mathrm{III}} \equiv \frac{\bar{c}_{\mathrm{E}_{\mathrm{C}}} \bar{c}_{\mathrm{cL}}+\bar{c}_{\mathrm{EL}}}{\bar{c}_{\mathrm{E}}}=\frac{K_{1} K_{3}}{1+K_{3}}
$$

with $\bar{c} \times$ defined as the equilibrium concentration of species $\mathrm{X}$.

For the more complex situation in Scheme II, the total bound ligand $B_{L}$ is given by eq 3 .

$$
B_{\mathrm{L}}=c_{\mathrm{L}}{ }^{i 01}-\bar{c}_{\mathrm{L}}=\frac{c_{\mathrm{E}}{ }^{\text {init }}}{\left[1+\frac{\Delta V}{V}\right]}\left(\frac{1}{1+\frac{K_{1}}{\bar{c}_{\mathrm{L}}}}+\frac{1}{1+\frac{K_{2}}{\bar{c}_{\mathrm{L}}}}\right)
$$


Equation 3 may be rearranged in several ways to permit analysis by means of a reciprocal plot. One rearrangement, suggested by its analogy to eq 1 , is given in eq 4

$$
\begin{aligned}
& \frac{\phi}{f B^{\prime}} \equiv \frac{\phi}{\left[1+\frac{\Delta V}{V}\right]\left[f_{\mathcal{C}_{\mathrm{L}}}{ }^{\mathrm{tot}}-F\right]}= \\
& \frac{1}{f_{\mathcal{C}_{\mathrm{E}}}^{\text {init }}}\left(1+\frac{f \phi}{F\left[\frac{1}{K_{1}}+\frac{1}{K_{2}}\right]}\right)
\end{aligned}
$$

where

$$
\phi=\frac{\left(K_{1}+K_{2}+2 \bar{c}_{\mathrm{L}}\right)\left(K_{1}+K_{2}\right)}{K_{1}^{2}+K_{2}^{2}+\bar{c}_{\mathrm{L}}\left(K_{1}+K_{2}\right)}
$$

Equation 4 is particularly useful when $K_{2} \gg K_{1}$ and $K_{2}>\bar{c}_{\mathrm{L}}$, in which case $\phi \simeq\left(K_{2}+2 \bar{c}_{\mathrm{L}}\right) /\left(K_{2}+\bar{c}_{\mathrm{L}}\right)$ and does not deviate greatly from 1. If preliminary estimates of $K_{1}$ and $K_{2}$ can be made, $\phi$ can be calculated for each experimental point and $\phi / f B^{\prime}$ can be plotted vs. $\phi / F$ to obtain precise values for $c_{\mathrm{E}}{ }^{\text {init }}$ and $K_{1}$.

Given values for $c_{E}$ init and $K_{1}$ from the titration analysis, $\bar{c}_{E}$ in Scheme I and $\bar{c}_{\mathrm{E}}$ corresponding to site 1 in Scheme II' can be calculated from eq 6 .

$$
\bar{c}_{\mathrm{E}}=\frac{K_{1} c_{E^{\text {init }}}}{\left(K_{1}+\bar{c}_{L}\right)(1+\Delta V / V)}
$$

Equation 6 also holds for Scheme III if $K_{1}$ is replaced by $K_{\text {II1. }}$.

Relaxation Times. Expressions relating the observed relaxation times $\tau_{1}$ and $\tau_{11}$ to the intrinsic rate constants in Schemes I-III have been given by Eigen and DeMaeyer (1963). For Scheme I, eq 7 obtains.

$$
\tau_{]}^{-1}=\tau_{1}^{-1} \equiv k_{12}\left(\bar{c}_{\mathrm{E}}+\bar{c}_{\mathrm{L}}\right)+k_{21}
$$

The observed $\tau_{1}^{-1}$ is equal to the intrinsic variable $\tau_{1}{ }^{-1}$ defined by eq 7 . Schemes II' $^{\prime}$ and III involve coupling through a species which participates in both reactions. To examine the effect of coupling in Scheme II' we define $\tau_{2}^{-1} \equiv k_{12}{ }^{\prime}\left(\bar{c}_{\mathrm{E}^{\prime}}+\overline{\mathrm{c}}_{\mathrm{L}}\right)+k_{21}{ }^{\prime}$, the reciprocal relaxation time for the second reaction in Scheme II' if it were completely uncoupled. One general condition which must obtain in Scheme $\mathrm{II}^{\prime}$ is given in eq 8 .

$$
\tau_{\mathrm{I}}^{-1} \tau_{\mathrm{II}}{ }^{-1}=\tau_{1}^{-1} \tau_{2}{ }^{-1}-k_{12} \bar{c}_{\mathrm{E}} k_{12}{ }^{\prime} \bar{c}_{\mathrm{E}^{\prime}}
$$

The analysis of coupled reactions is greatly facilitated if one relaxation time is much greater than the other. This is the case for all the relaxation measurements in this report; the faster relaxation is at least eight times the slower in every instance. If $\tau_{I I}^{-1} \gg \tau_{I}^{-1}$, then we ascribe $\tau_{11}$ to the second binding site in Scheme II' and assume $\tau_{11}{ }^{-1} \simeq \tau_{2}{ }^{-1}$. Appropriate substitution into eq 8 gives eq 9

$$
\tau_{\mathrm{I}}^{-1} \simeq k_{12}\left(\bar{c}_{\mathrm{E}} \theta+\bar{c}_{\mathrm{L}}\right)+k_{21}
$$

where $\theta^{-1} \equiv\left[1+\bar{c}_{E^{\prime}} /\left(K_{2}+\bar{c}_{\mathrm{L}}\right)\right]$. The term $\theta$ is a measure of the coupling between the two reactions; coupling becomes negligible as $\theta \rightarrow 1$.

The application of eq 7 and 9 in this study is referred to as method $\mathrm{A}$ and presumes that $\bar{c}_{\mathrm{L}}, c_{\mathrm{E}}{ }^{\text {init }}, K_{1}$, and $K_{2}$ are known parameters and that $\bar{c}_{E}$ is obtained from eq 6 . Plots of $\tau_{I}^{-1}$ vs. $\left(\bar{c}_{\mathrm{E}}\left(\right.\right.$ or $\left.\left.\bar{c}_{\mathrm{E}} \theta\right)+\bar{c}_{\mathrm{L}}\right)$ yield $k_{12}$ as the slope and $k_{21}$ as the $\tau_{\mathrm{I}}^{-1}$ axis intercept.

An alternative form of relaxation time analysis, based on a report by Eigen and Winkler-Oswatitsch (1977), is being introduced in this report. This analysis, called method B, avoids calculations of equilibrium concentrations and instead requires only that $c_{L}{ }^{\text {tot }}$ be known and that $c_{E}{ }^{\text {tot }}$, the total enzyme concentration, be constant. For Scheme I, the equilibrium concentrations in eq 7 can be expressed in terms of $c_{\mathrm{L}}{ }^{\text {tot }}$ and $c_{\mathrm{E}}{ }^{\text {tot }}$ as shown in eq 10 .

$$
\begin{aligned}
\tau_{1}^{-2}=k_{12}{ }^{2}( & \left.K_{1}+c_{E}{ }^{\mathrm{tot}}\right)^{2}+2 k_{12}{ }^{2}\left(K_{1}-c_{E}{ }^{\mathrm{tot}}\right) c_{\mathrm{L}}{ }^{\mathrm{tot}} \\
& +k_{12}{ }^{2}\left(c_{\mathrm{L}}{ }^{\mathrm{tot}}\right)^{2}=A+B c_{\mathrm{L}}{ }^{\mathrm{tot}}+C\left(c_{\mathrm{L}}{ }^{\mathrm{tot}}\right)^{2}
\end{aligned}
$$

This second-order polynomial can be analyzed by least-squares regression analysis to give the best-fit values of $A, B$, and $C$ which, in turn, can be converted to $k_{12}, K_{1}$, and $c_{E}{ }^{\text {tot }}$. Unfortunately, eq 10 and Scheme $I$ are insufficient for the analysis of some of our data. Equation 10 can be modified to apply to Scheme II' and to include a small dilution correction factor under certain conditions. In particular, if we define the constant $\theta_{0}$ as the limit of $\theta$ (eq 9 ) as $\bar{c}_{1} \rightarrow 0$ and assume that $K_{2} \gg \bar{c}_{1}$. such that $\theta \simeq \theta_{0}$ for the entire data set, eq 10 can be applied to Scheme II'. Furthermore, if $\Delta V / V$ (eq $1 \mathrm{ff}$ ) is both approximately proportional to $\mathrm{C}_{\mathrm{L}}{ }^{\text {tot }}$ and small enough that $(1+$ $\Delta V / V)^{-1} \simeq 1-\Delta V / V$, then coefficients $A, B$, and $C$ in eq 10 applied to Scheme II' are given by eq 11-13.

$$
\begin{gathered}
A \simeq k_{\text {on }}{ }^{2}\left(K_{I I}+c_{E} E^{\text {init }}\right)^{2} \\
B \simeq 2 k_{\text {on }}{ }^{2}\left(K_{\mathrm{II}}-c_{\mathrm{E}}{ }^{\text {init }}\left[1+\rho\left(c_{\mathrm{E}}{ }^{\text {init }}+K_{\mathrm{H}}\right)\right]\right) \\
C \simeq k_{\mathrm{on}}{ }^{2}\left(1+\rho c_{\mathrm{E}}^{\text {init }}\right)^{2}
\end{gathered}
$$

where $k_{\text {on }} \equiv k_{12} \theta_{0}, K_{\mathrm{Il}} \boldsymbol{\nabla} K_{1} / \theta_{0}$, and $\rho \equiv \Delta V / c_{\mathrm{L}}{ }^{\text {tot }} V$. If $\theta_{0}$ and $\rho$ can be estimated independently, $K_{1}, c_{\mathrm{E}}{ }^{\text {init }}$, and $k_{12}$ can be obtained from eq 11-13 by successive approximations.

Competitive Inhibition. In the presence of a competitive inhibitor I, Scheme I is extended to Scheme IV.

SCHEME IV

$$
\mathrm{E}+\mathrm{L} \underset{k_{21}}{\stackrel{k_{12}}{\rightleftharpoons}} \mathrm{EL} \quad \mathrm{E}+\mathrm{I} \underset{k_{-\mathrm{i}}}{\stackrel{k_{+1}}{\rightleftharpoons}} \mathrm{EI}
$$

The formal treatment of Scheme IV is identical to that of Scheme II', although the coupling occurs through $E$ rather than L. Thus, if $\tau_{i}^{-1} \bullet\left[k_{\mathrm{i}}\left(\bar{c}_{\mathrm{E}}+\bar{c}_{1}\right)+k_{-\mathrm{i}}\right] \gg \tau_{1}^{-1}, K_{\mathrm{i}} \gg \bar{c}_{\mathrm{E}}$ and $\theta_{0}^{-1} \equiv\left(1+\bar{c}_{1} / K_{\mathrm{i}}\right)$ where $K_{\mathrm{i}}=k_{-\mathrm{i}} / k_{\mathrm{i}}$, eq 14 obtains for the observed relaxation time $\tau_{\text {obsd }}$.

$$
\tau_{\text {obsd }}{ }^{-1}=k_{\text {on }}\left(\bar{c}_{E}+\bar{c}_{\mathrm{El}}+\bar{c}_{\mathrm{L}}+K_{\mathrm{obsd}}\right)
$$

where $k_{\text {on }} \equiv k_{12} \theta_{0}$ and $K_{\text {obsd }} \equiv K_{1} / \theta_{0}$. Equilibrium titration can provide $K_{\text {obsd }}$ (eq 1 holds for Scheme IV if $K_{\text {obsd }}$ replaces $K_{1}$ ) and $\bar{c}_{\mathrm{E}}+\bar{c}_{\mathrm{EI}}$ (eq 6 holds for Scheme IV if $\bar{c}_{\mathrm{E}}+\bar{c}_{\mathrm{EI}}$ replaces $\bar{c}_{\mathrm{E}}$ and $K_{\text {obsd }}$ replaces $K_{1}$ ). Substitution of these terms into eq 14 allows the determination of $k_{\text {on }}$ from relaxation data. Alternatively, $k_{\text {or }}$ and $K_{\text {obsd }}$ can be obtained from the second-order polynomial analysis in eq 10-13.

Ligand Protonation. 1-Methyl-7-hydroxyquinolinium has a $\mathrm{p} K_{\mathrm{a}}$ of 5.9 (Rosenberry and Bernhard, 1971). Data in the Results indicate that only the protonated ligand binds. In this case, Scheme V is assumed.

SCHEME $\checkmark$

$$
\mathrm{L}+\mathrm{H}^{+} \underset{k_{-a}}{\stackrel{k_{\mathrm{a}}}{\rightleftharpoons}} \mathrm{LH}^{+} \quad \mathrm{LH}^{+}+\mathrm{E} \underset{k_{21}}{\stackrel{k_{12}}{\rightleftharpoons}} \mathrm{E} \cdot \mathrm{LH}^{+}
$$

Formal treatment of Scheme $\mathrm{V}$ is similar to that for Scheme II'. Because protons in the solution are buffered, $\mathrm{d} \vec{c}_{\mathrm{H}} / \mathrm{d} t=0$. If the proton equilibration with $\mathrm{L}$ is rapid, $\tau_{\mathrm{a}}^{-1} \equiv\left[k_{\mathrm{a}} \bar{c}_{\mathrm{H}}+k_{-\mathrm{i}}\right]$ $\gg \tau_{1}^{-1}$ and eq 15 holds

$$
\tau_{\text {obsd }}{ }^{-1}=k_{\text {on }}\left(\bar{c}_{\mathrm{E}}+\bar{c}_{\mathrm{L}}+\bar{c}_{\mathrm{LH}}+K_{\text {obsd }}\right)
$$


where $k_{\text {on }}=k_{12} \theta_{0}, \theta_{0}^{-1}=\left(1+K_{\mathrm{a}} / \bar{c}_{\mathrm{H}}\right), K_{\mathrm{a}}=k_{-\mathrm{a}} / k_{\mathrm{a}}$, and $K_{\text {obsd }}=K_{1} / \theta_{0}$. Treatment is similar to that following eq 14 .

Relaxation Amplitudes. The total amplitude $\Delta F_{\text {tot }}$ of the one-step process in Scheme I has been derived by Eigen and DeMaeyer (1963) and is given by eq 16

$$
\Delta F=\left[\frac{f \bar{c}_{\mathrm{E}} \bar{c}_{\mathrm{L}}}{\bar{c}_{\mathrm{E}}+\bar{c}_{\mathrm{L}}+K}\right]\left[\frac{\Delta H \Delta T}{R T^{2}}\right]
$$

where $\Delta F \equiv \Delta F_{\text {tot }} \equiv \Delta F_{\mathrm{I}}, K \equiv K_{1}$, and $\Delta H \equiv \Delta H_{1}$, the standard enthalpy difference between products and reactants. The second term on the right of eq 16 is constant for a given temperature change $\Delta T$. General expressions for the relaxation amplitudes in the two-step process in Scheme II' have been derived by Thusius (1972). For the case where $\tau_{\mathrm{II}}{ }^{-1} \simeq \tau_{2}{ }^{-1}$ $\gg \tau_{\mathrm{I}}^{-1}$ (see eq $8 \mathrm{ff}$ ), $\Delta F_{\mathrm{II}}=\Delta F_{2}$; and $\Delta F_{2}$ is given by eq 16 with $K \equiv K_{2}$ and $\Delta H \equiv \Delta H_{2}$. In this case, the amplitude of the slower step $\Delta F_{\mathrm{I}}$ is given by eq 17

$$
\begin{aligned}
& \Delta F_{\mathrm{I}} \simeq\left[\frac{f \bar{c}_{\mathrm{E}} \theta \bar{c}_{\mathrm{L}}}{\bar{c}_{\mathrm{E}} \theta+\bar{c}_{\mathrm{L}}+K_{\mathrm{I}}}\right] \\
& \times\left[\Delta H_{1}+(1-\theta) \Delta H_{2}\right]\left[\frac{\Delta T}{R T^{2}}\right]
\end{aligned}
$$

where $\theta$ is given below eq 9 . The second and third terms on the right are virtually constant if $\theta$ is both approximately constant and close to 1 , the case in the current data.

In Scheme III the two observed relaxation processes are most appropriately considered in terms of a single overall equilibrium with $\Delta F_{\text {tot }}=\Delta F_{1}+\Delta F_{11}$. If it is assumed that only the free ligand fluoresces, then eq 16 obtains with $\Delta F \equiv \Delta F_{\text {tot }}$, $K \boxminus K_{\text {III }}($ eq 2$)$, and $\Delta H \equiv\left(\Delta \ln K_{\mathrm{III}}\right)\left(R T^{2}\right) /(\Delta T){ }^{1}$

Data Analysis. Analyses of linear functions were carried out with the use of a weighted least-squares computer procedure as described previously (Rosenberry and Bernhard, 1971). Data points of the dependent variable $y$ were weighted by the reciprocal of their estimated variance, Var $y$; these estimates either (1) assumed constant percent error (Var $y / y^{2}$ was a constant for the entire data set) or (2) were calculated directly when multiple observations were made. Data points of the independent variable $x$ are required to be invariant in this analysis.

Analyses of second-order polynomials (eq 10) were made by a weighted least-squares procedure for polynomials of order $n$ (Forsythe, 1957; Kelly, 1967). The procedure used regression analysis computer subroutine programs RLFOTW and RLDOPM supplied by IMSL (International Mathematical and Statistical Libraries, Houston, Texas), which calculated the coefficients $A, B$, and $C$ of eq 10 . Initial estimates of the reaction parameters were obtained from the following rearrangements of eq $10: k_{12}=(C)^{1 / 2} ; C_{E^{t o t}}=\left[(A / C)^{1 / 2}-B /\right.$ $2 C] / 2 ; K_{1}=\left[(A / C)^{1 / 2}+B / 2 C\right] / 2$. Final estimates of reaction parameters utilized these initial estimates in the following rearrangements of eq $11-13$ : let $C^{*}=C /\left(1+\rho c_{\mathrm{E}}^{\text {tot }}\right)^{2}$; then $k_{\text {on }}=\left(C^{*}\right)^{1 / 2} ; c_{E^{\text {init }}}=\left[\left(A / C^{*}\right)^{1 / 2}-B / 2 C^{*}\right] /\left[2+\rho\left(c_{E^{\text {tot }}}+\right.\right.$ $\left.\left.K_{1}\right)\right] ; K_{\mathrm{II}}=\left(A / C^{*}\right)^{1 / 2}-C_{\mathrm{E}}{ }^{\text {init }}$. It is obvious that variance estimates of most of these reaction parameters require estimates of the covariances of $A$ and $C$ and $B$ and $C$. Covariance estimates programs were not yet available from IMSL. Consequently, variance estimates of $k_{12}, K_{1}$, and $c_{E^{\text {init }}}$ in Table I

1 This application of eq 16 to Scheme III derives from the easily demonstrated equality

$$
\begin{array}{r}
\Delta F_{\mathrm{lOt}}=f\left[\frac{1}{\bar{c}_{\mathrm{E}}}+\frac{1}{\bar{c}_{\mathrm{L}}}+\frac{1}{\bar{c}_{\mathrm{EL}}+\bar{c}_{\mathrm{EL}}}\right]^{-1}\left[\Delta \ln K_{1}+\frac{\Delta \ln K_{2}}{1+K_{2}}\right] \\
=\frac{f \bar{c}_{\mathrm{E}} \bar{c}_{\mathrm{L}}}{\bar{c}_{\mathrm{E}}+\bar{c}_{\mathrm{L}}+K_{\mathrm{III}}} \Delta \ln K_{\mathrm{III}}
\end{array}
$$

TABLE I: Thermodynamic and Kinetic Constants Observed for the Interaction of $N$-Methylacridinium and Acetylcholinesterase in 0.1 M Sodium Phosphate, $\mathrm{pH} 8.0$ at $23^{\circ} \mathrm{C}$. $^{a}$

\begin{tabular}{ccc}
\hline Method & $\begin{array}{c}K_{1} \\
(\mu \mathrm{M})\end{array}$ & $\begin{array}{c}k_{12} \\
\left(\mathrm{nM}^{-1} \mathrm{~s}^{-1}\right)\end{array}$ \\
\hline $\mathrm{A}$ & $0.149 \pm 0.003$ & $1.18 \pm 0.03$ \\
$\mathrm{~B}^{b}$ & $0.103 \pm 0.012$ & $1.23 \pm 0.05$ \\
\hline
\end{tabular}

a Method $\mathrm{A}$ involves separate determinations of $K_{1}$ and $k_{12}$ according to eq 4 and 9 . In Method $\mathrm{B}, K_{1}$ and $k_{12}$ are evaluated simultaneously according to eq 10 through 13 (see Figure 5). Data are from Figures 2, 4, and 5. ${ }^{b}$ Estimates utilize $\rho=0.00225$ and $\theta_{0}{ }^{-1}=$ 1.140 .

(Method B) and Figure 5 were made experimentally by analysis of ten random data sets generated by the equation $\tau_{\mathrm{iR}}=$ $\bar{\tau}_{i}+\left(\operatorname{Var} \bar{\tau}_{i}\right)^{1 / 2}(R)$ where $\bar{\tau}_{i}$ is an observed mean relaxation time, $R$ is a random Gaussian number from a Gaussian distribution of standard deviation equal to 1 , and $\tau_{\mathrm{iR}}$ is the generated mean relaxation time.

All listed error estimates are standard deviations of the mean parameter estimate, defined as the square root of the variance of the mean parameter estimate.

\section{Results}

Relaxation Spectra Associated with N-Methylacridinium and Acetylcholinesterase. Relaxation measurements were carried out in $0.1 \mathrm{M}$ sodium phosphate for two reasons. (1) The fluorescence of $N$-methylacridinium was quenched by about a factor of 2 in $0.1 \mathrm{M} \mathrm{NaCl}$ relative to $0.1 \mathrm{M}$ sodium phosphate. A Stern-Volmer analysis indicated that quenching arose from a complex between $N$-methylacridinium and $\mathrm{Cl}^{-}$. A rapid relaxation was observed when a mixture of $N$-methylacridinium and $\mathrm{Cl}^{-}$was perturbed in temperature-jump experiments. Because this relaxation was coupled to that involving ligand association with the enzyme (a situation formally identical to Scheme II'), subsequent experiments avoided $\mathrm{Cl}^{-}$. (2) A complicated relaxation spectrum was observed for the interaction of either $N$-methylacridinium or 1-methyl-7-hydroxyquinolinium with acetylcholinesterase at $\mathrm{pH} 6.0$ and 5.3. Because the spectrum was greatly simplified at higher $\mathrm{pH}$ values, the complicated spectrum at or below pH 6.0 suggests the involvement of both protonated and unprotonated enzyme forms in the binding of cationic ligands. This topic is beyond the scope of the present paper. However, it was decided to work at $\mathrm{pH}$ 8.0-8.5 where possible to avoid a contribution due to protonated enzyme forms. At this $\mathrm{pH}, 0.1 \mathrm{M}$ sodium phosphate is required for sufficient buffering capacity.

Typical relaxation spectra obtained with $N$-methylacridinium and acetylcholinesterase at $\mathrm{pH} 8.0$ are shown in Figure 1. At the relatively low ligand concentration in Figure $1 \mathrm{a}$, the sudden temperature increase of about $3^{\circ} \mathrm{C}$ gives rise to a relatively large increase in fluorescence; the overall fluorescence increase or total relaxation amplitude $\Delta F_{\text {tot }}$ corresponds to nearly $15 \%$ of the total fluorescence $F$ at these ligand concentrations. This fluorescence increase can be fit precisely to a single exponential curve and thus can be characterized by a single relaxation time. At higher ligand and high enzyme concentrations as in Figure $1 \mathrm{~b}, \Delta F_{\text {tot }} / F$ decreases and two distinct relaxation steps become apparent. The fast step is displayed with a decreased time scale in Figure 1c. The relaxation time of $33 \mu$ s calculated for this fast step can be clearly distinguished from the relaxation time or rise time associated with the temperature-jump forcing function itself. This rise time is shown in Figure 1d, where a temperature jump is ap- 

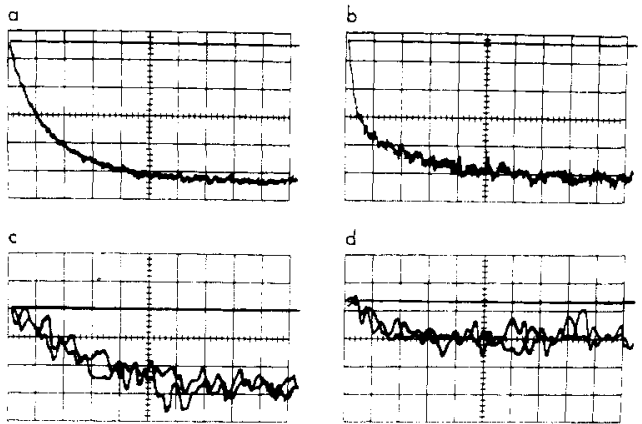

FIGURE 1: Relaxation spectra of $N$-methylacridinium and acetylcholinesterase in $0.1 \mathrm{M}$ sodium phosphate at $\mathrm{pH} 8.0$ and $23^{\circ} \mathrm{C}$. The total fluorescence $F$ was adjusted to $20 \mathrm{~V}$ in each experiment, and, in spectra a-c. $c^{\prime} \mathrm{E}^{\text {init }}=2.92 \mu \mathrm{M}$. (a) $c_{\mathrm{L}}{ }^{\mathrm{tot}}=2.28 \mu \mathrm{M}, \overline{\mathrm{c}}_{\mathrm{L}}=0.31 \mu \mathrm{M} ;-0.5 \mathrm{~V} /$ div vertically, $0.5 \mathrm{~ms} /$ div horizontally. (b) $c^{\text {tot }}=4.84 \mu \mathrm{M}, \bar{c}_{1}=1.94 \mu \mathrm{M} ;-0.1$ $\mathrm{V} / \mathrm{div}, 0.2 \mathrm{~ms} / \mathrm{div}$. (c) $c_{\mathrm{L}} \mathrm{L}^{\mathrm{tot}}=5.91 \mu \mathrm{M}, \bar{c}_{\mathrm{L}}=2.91 \mu \mathrm{M} ;-0.1 \mathrm{~V} / \mathrm{div}, 10$ $\mu \mathrm{s} /$ div. (d) $c_{L}{ }^{\text {tot }}=\bar{c}_{L}=3.15 \mu \mathrm{M} ; c_{\mathrm{E}}{ }^{\text {init }}=0 ;-0.1 \mathrm{~V} / \mathrm{div}, 10 \mu \mathrm{s} / \mathrm{div}$. In $\mathrm{c}$ and $d$ two temperature jumps are superimposed.

plied to a ligand solution in the absence of enzyme. A rise time of $8 \mu \mathrm{s}$ and a $\Delta F_{\text {tot }} / F=0.0064$ is observed for this control condition; this intrinsic change in ligand fluorescence was subtracted as the $\Delta F$ values for mixtures of ligand and enzyme were obtained.

Three or four temperature-jump measurements were made at each $\bar{c}_{L}$ value. At lower $\bar{c}_{L}$ (e.g., the first six points in Figure $5)$, the standard deviation of the mean slow relaxation time was $<5 \%$ of the mean. At higher $c_{\mathrm{L}}$, the standard deviation was $<10 \%$ of the mean slow relaxation time. An insufficient number of fast relaxation times were measured for adequate statistical analysis.

It should be noted that the fast relaxation step is observed only at high enzyme normalities $(>1 \mu \mathrm{M})$. At lower enzyme normalities the amplitude of this step becomes vanishingly small.

Equilibrium Titration of Acetylcholinesterase with $N$ Methylacridinium. A quantitative evaluation of the relaxation data conventionally requires observation or calculation of both the equilibrium free ligand concentration $\vec{c}_{L}$ and the equilibrium free enzyme concentration $\bar{c}_{\mathrm{E}}$. An equilibrium titration of acetylcholinesterase with $N$-methylacridinium is displayed as a Scatchard plot in Figure 2. This titration was carried out simultaneously with relaxation measurements by sequential additions of ligand to the temperature-jump cell. The ligand fluorescence $F$ was measured both prior to and after the relaxation measurements at a given total ligand concentration (1 $1^{\text {tot }}$. At each $c_{1}^{\text {tot }}$ the initial $F$ was slightly greater than the final $F$. This progressive loss of fluorescence amounted to about $2 \%$ at each $\mathrm{c}_{\mathrm{l}}{ }^{\text {tut }}$. The loss was less pronounced at lower $\mathrm{pH}$ and more pronounced at higher $\mathrm{pH}$ in the absence of $\mathrm{Ca}^{2+}$ (see below). We assumed that this fluorescence decrease represented a net loss of ligand from the system (see Discussion) and applied a cumulative correction to $c_{\mathrm{L}}{ }^{10 t}$ for the observed fluorescence loss in the temperature-jump cell.

The data in Figure 2 conform to a single-binding isotherm at low ligand concentrations, but a second, lower affinity binding site is also suggested at the highest ligand concentrations. The relaxation amplitude analysis described in the following section independently confirmed the presence of a second ligand-binding site, and final analysis of the data in Figure 2 was based on Scheme II'.

Inclusion of the correction for the second, low-affinity binding site was important to a quantitative characterization of the relaxation data at $c_{\mathrm{E}}{ }^{\mathrm{init}}>1.0 \mu \mathrm{M}$. For the initial estimate of $K_{1}$, in which Scheme I was assumed to apply to the first eight

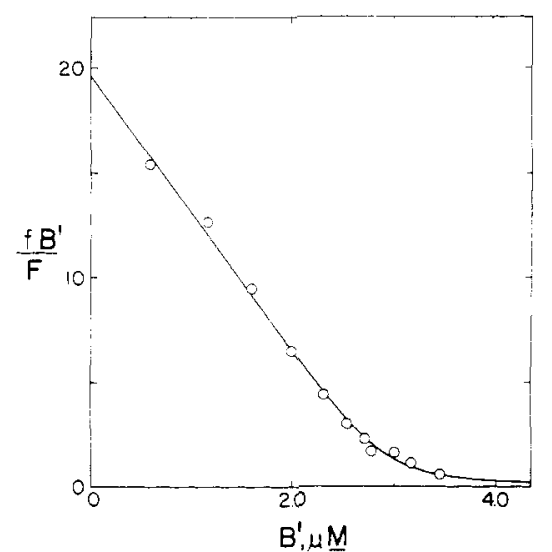

FiGURE 2: Equilibrium titration of acetylcholinesterase with $N$-methylacridinium. Experimental conditions are given in Table I. Data were quantitatively analyzed by the reciprocal plots in eq 1 and 4 , but are displayed here as a Scatchard plot for additional clarity. Assuming a single high-affinity site as in Schemes I or III, an initial estimate of $K_{1}$ or $K_{111}$ was made with the use of the eight points at the lowest ligand concentration. When relaxation amplitude analyses (Figure 3 ) indicated two independent binding sites (Scheme $\mathrm{II}^{\prime}$ ), all eleven data points were reanalyzed according to eq 4 , with $\phi / F$ the independent variable and $\phi / B^{\prime}$ the dependent variable. $\phi$ was calculated according to eq 5 with $K_{1}=0.15 \mu \mathrm{M}$ and $K_{2}=20 \mu \mathrm{M}$. The final estimates were $K_{1}=0.149 \pm 0.003 \mu \mathrm{M}$ and $c^{i m i i}=2.92 \pm 0.02 \mu \mathrm{M}$. The line was calculated from eq 3 using the final estimates for $K_{\mid}$and $c_{\mathrm{F}}$ init and $K_{2}=20 \mu \mathrm{M}$.

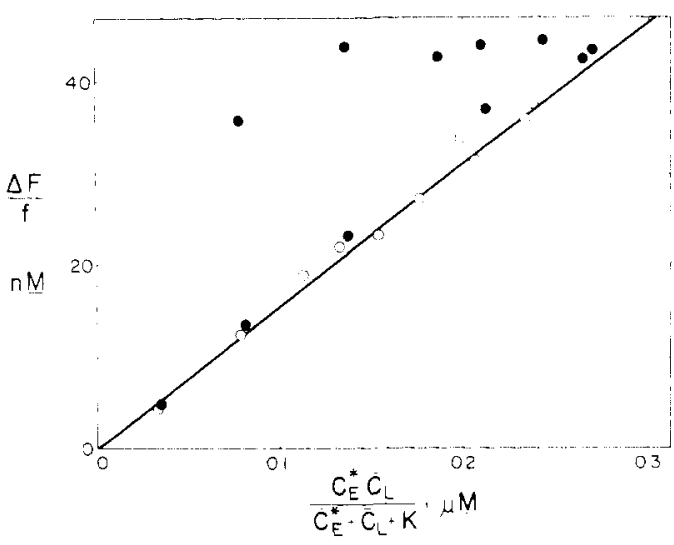

FIGURE 3: Analysis of the mean relaxation amplitudes observed with acetylcholinesterase and $N$-methylacridinium. Two analyses which correspond to Schemes II' and III are shown, as described in the text. Data correspond to the experiment in Figure $2 ; c_{E}{ }^{i n i}=2.92 \mu \mathrm{M}$. (O) Scheme $\mathrm{II}^{\prime}$ according to eq $17 ; \Delta F \equiv \Delta F_{\mathrm{l}}, K \equiv K_{1}, \bar{c}_{\mathrm{E}}{ }^{*} \equiv \bar{c} \mathrm{E} \theta$, where $K_{\mathrm{l}}, K_{2}$, and $\bar{c}_{E}$ reflect the final estimates from Figure 2 and $\theta$ is calculated independently for each data point assuming $C_{E}{ }^{\text {init }}=C_{E}{ }^{\text {init }}$. The solid line is calculated from a least-squares analysis of these points assuming constant percent error with the addition of virtually infinite weight at the origin (-) Scheme III: $\Delta F \equiv \Delta F_{\text {tot }}, K \equiv K_{\text {III }}$, where $K_{\text {III }}$ (eq 2) and $\bar{c}_{\mathrm{E}}{ }^{*} \equiv \bar{c}_{\mathrm{E}}$ rellect the initial estimates from Figure 2.

points in Figure 2, $K_{1}$ was $5 \%$ less and $c_{E}{ }^{\text {init }} 5 \%$ more than the final estimates assuming Scheme $\mathrm{II}^{\prime}$. Corresponding $\bar{c} \mathrm{E}$ estimates (eq 6) using these initial values were 6 to $10 \%$ less than the final estimates, and the experimental point scatter in Figures 3 and 4 below was significantly improved when the final $\bar{c}_{\text {l: }}$ estimates replaced the initial estimates.

Analysis of Relaxation Amplitudes. The observation of two relaxation processes at high enzyme normality in Figure 1 implies the presence of at least two equilibria in the interaction of $N$-methylacridinium with acetylcholinesterase. Schemes II' and III represent two of the simplest systems which involve two equilibria. While the equilibrium titration data in the previous section suggest that Scheme II' obtains, we examined the relaxation data to see if it would independently confirm 


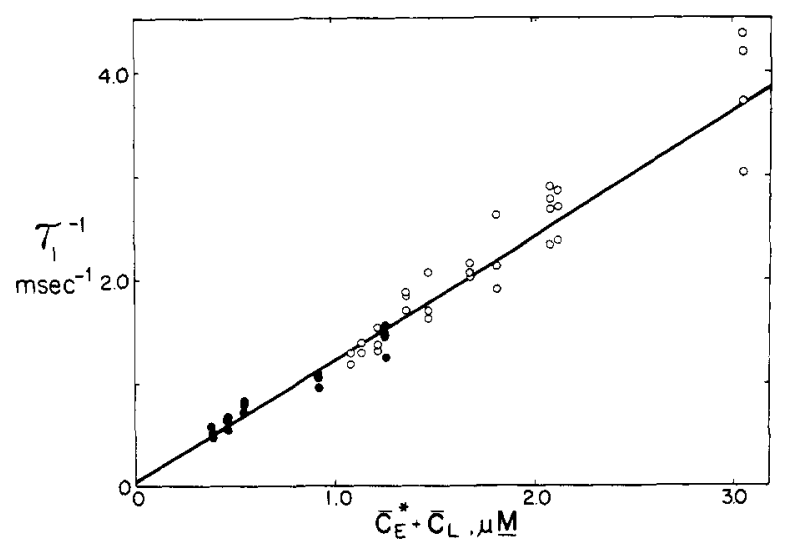

FIGURE 4: Dependence of the relaxation times observed with acetylcholinesterase and $N$-methylacridinium on equilibrium concentrations of the reactants. Data are plotted according to eq 9 , where $C_{E} *=\bar{c}_{E} \theta$. (O) Reciprocal relaxation times correspond to the experiments in Figures 2 and 3; $c_{\mathrm{E}}{ }^{\text {init }}=2.92 \mu \mathrm{M}$; and $\theta$ for each data point is identical to that for the corresponding experiment in Figure 3. (•) Data from a different stock enzyme dilution obtained on a nother day; $C_{\mathrm{E}}^{\text {init }}=0.49 \mu \mathrm{M}$. The line was calculated from a least-squares analysis. In this analysis, mean $\tau_{1}^{-1}$ values were calculated at each discrete $\left(\bar{c}_{\mathrm{E}}^{*}+\bar{c}_{\mathrm{L}}\right)$ condition, and each mean was weighted by the reciprocal of its variance. The line corresponds to $k_{12}=$ $1.18 \pm 0.03 \times 10^{9} \mathrm{M}^{-1} \mathrm{~s}^{-1}$.

Scheme II' and reject Scheme III. The relatively small relaxation time $\tau_{11}$ associated with the fast step made its analysis in terms of $\bar{c}_{\mathrm{E}}$ and $\bar{c}_{\mathrm{L}}$ rather imprecise. The relaxation amplitude of this step was obtained with greater precision. An analysis of relaxation amplitudes offers the further advantage that, when $\tau_{I I}^{-1} \gg \tau_{1}^{-1}$, a clear distinction between Schemes II' and III can be made without assumptions about the intrinsic rate constants for the two observed steps. Thus, for Scheme III, with the restriction that only the free ligand fluoresces, the total relaxation amplitude $\Delta F_{\text {tot }}$ follows the simple concentration dependence given by eq $16 \mathrm{ff}$, while, for Scheme $\mathrm{II}^{\prime}$, the relaxation amplitude $\Delta F_{1}$ (slow step) is given by eq 17. In Figure 3 , amplitude analyses corresponding to these two alternatives are shown. A much better fit of the data is obtained when $\Delta F_{\mathrm{I}}$, rather than $\Delta F_{\text {tot }}$, is the dependent variable. Thus, Scheme II', but not Scheme III, is consistent with the data at high enzyme normalities. ${ }^{2}$ Below $c_{\mathrm{E}}{ }^{\text {init }}$ values of $1.0 \mu \mathrm{M}, \theta$ values in Scheme II' are greater than 0.95 and Scheme I is an excellent approximation.

From the slope of the line in Figure 3, $\Delta H_{1}$ may be approximated (eq 17) with the assumption that $(1-\theta) \simeq 0$. (Assuming $K_{2}=20 \mu \mathrm{M}, \theta$ varies between 0.88 and 0.91 .) For the $3.3^{\circ} \mathrm{C}$ temperature change, $\Delta H_{1} \simeq 34.3 \mathrm{~kJ} / \mathrm{mol}(8.2$ $\mathrm{kcal} / \mathrm{mol}$ ).

\footnotetext{
2 Although equilibrium titration data indicate that the fluorescence of bound ligand is $\leqslant 0.1 \%$ of the free ligand fluorescence, it could be argued that within Scheme III a slightly fluorescent EL is present in low concentration relative to nonfluorescent EL*. If the fast relaxation observed here then corresponded to the $K_{3}$ equilibrium in such a Scheme III', the amplitude of the slow step $\Delta F_{1}$ would be given by eq 18 (see Thusius, 1972)$$
\Delta F_{I} \cong\left[\frac{\bar{c}_{\mathrm{E}} \bar{c}_{\mathrm{L}}}{\bar{c}_{\mathrm{E}}+\bar{c}_{\mathrm{L}}+K_{\mathrm{III}}}\right]\left[\Delta \ln K_{\mathrm{III}}\right]\left[\left(f_{\mathrm{L}}-f_{\mathrm{EL}}\right)-f_{\mathrm{EL}}\left(1+K_{3}\right)^{-1}\right]
$$

where $K_{l I}$ is defined in eq $2, f_{\mathrm{L}}$ is the fluorescence intensity coefficient for free ligand, and $f_{\mathrm{EL}}$ is the fluorescence intensity coefficient for ligand bound in the EL species. The predictions of eq 17 for Scheme $\mathrm{II}^{\prime}$ and of eq 18 for Scheme III' are very similar, and the amplitude data in Figure 3 cannot distinguish between them. However, Scheme III' can be rejected because it predicts that the relaxation times $\tau_{I I}$ for the fast step should be independent of $\bar{c}_{L}$ and $\bar{c}_{\mathrm{E}}$. In fact the relaxation times $\tau_{11}$ are observed to decrease about $30 \%$ as $\bar{c}_{\mathrm{L}}$ increases from 1.6 to $5.5 \mu \mathrm{M}$, a result consistent with Scheme II',
}

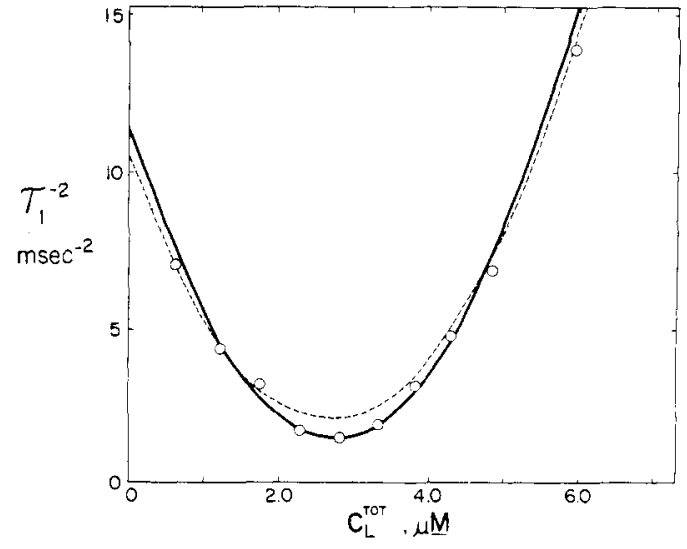

FIGURE 5: Dependence of the mean relaxation times observed with acetylcholinesterase and $N$-methylacridinium on the total ligand concentration. Mean relaxation times are identical to those in Figure 4, where $\mathcal{C}_{E}^{\text {init }}=2.92 \mu \mathrm{M}$ by equilibrium titration. Data are plotted according to eq 10 where the coefficients $A, B$ and $C$ are given by eq $11-13$ with the necessary assumption that $\theta \simeq \theta_{0}=0.877$. The dashed line was inserted with the use of calculated $A, B$, and $C$ values obtained from reaction parameter estimates from Figures 2 and 4 (method $A$, Table 1 ). The solid line was calculated from a weighted least-squares analysis which assumes a second-order polynomial in $C_{L}{ }^{\text {tot }}$ (see the Experimental Section). The solid line corresponds to the following values, obtained by simultaneous solution of eq 10-12: $k_{12}=1.23 \pm 0.05+10^{9} \mathrm{M}^{-1} \mathrm{~s}^{-1} ; K_{1}=0.103 \pm$ $0.012 \mu \mathrm{M}$, and $c_{\mathrm{E}}^{\text {init }}=2.94 \pm 0.04 \mu \mathrm{M}$.

Assuming Scheme $\mathrm{II}^{\prime}$, analysis of $\Delta F_{\mathrm{II}}$ permits an estimate of $K_{2}$. Trial values of $K_{2}$ were inserted in eq 16 to obtain the best correspondence to the $\Delta F_{\text {II }}$ data. The data were not highly precise, but $K_{2}=20 \mu \mathrm{M}$ could be estimated within a factor of two. This estimate was sufficiently accurate to allow calculation of $\phi$ values (eq 5) for use in the equilibrium titration data in Figure 2.

Analysis of Relaxation Times. The observed reciprocal relaxation times for the slow step $\left(\tau_{1}^{-1}\right)$ were plotted against the equilibrium concentrations in accordance with eq 9 , as shown in Figure 4. The calculated value for $k_{12}$ is given in Figure 4 and Table I (method A).

An independent analysis of the observed $\tau_{\text {I values was car- }}$ ried out assuming only $\mathcal{C}_{\mathrm{L}}{ }^{\text {tot }}$ as the independent variable. Data were analyzed with the use of eq 10 , and $c_{\mathrm{E}}^{\text {init }}, K_{1}$, and $k_{12}$ were obtained from eq 11-13 as outlined in the Experimental Section. This analysis is given in Figure 5 and Table I (method B), and a quite reasonable correspondence with the more conventional determination in Figure $4(\operatorname{method} A)$ was obtained despite the required slight approximation $\theta \simeq \theta_{0}$ in method $\mathrm{B}$. Methods $\mathbf{A}$ and $\mathbf{B}$ agree precisely on the value of $c_{\mathrm{E}}{ }^{\text {init }}$. This agreement is largely due to the condition $c_{\mathrm{E}}{ }^{\text {init }} \gg K_{1}$, thus allowing considerably greater accuracy in determining $C E^{\text {init }}$ than $K_{1}$ in method B. Indeed $K_{1}$ in method B is only about $70 \%$ that of the more accurately determined $K_{1}$ value in method $\mathrm{A}$ and has a much higher relative standard deviation. Nevertheless, the difference between $K_{1}$ in methods A and B is larger than that predicted by the variance of the mean relaxation times. This suggests either that the approximations underlying the use of method B (see eq $10 \mathrm{ff}$ ) or, more likely, that some systematic inconstancy, e.g., slight progressive loss of $E$ or $L$ from the system, is influencing the estimates. Estimates of $k_{12}$ are also listed in Table I, and methods $A$ and $B$ agree within the limits predicted by the experimental variance.

The dissociation rate constant $k_{21}$ may be obtained directly from the $\tau_{1}{ }^{-1}$ intercept in Figure 4 or as the product of $K_{1}$ and $k_{12}$ in Table I. Because $c_{\mathrm{E}}^{\text {init }} \gg K_{1}$, the intercept in Figure 4 is too near the origin for an accurate estimate of $k_{21}$. However,

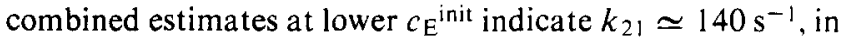


TABLE II: $\mathrm{Ca}^{2+}$ as an Inhibitor of the Binding of

$V$-Methylacridinium to Acetylcholinesterase in $0.1 \mathrm{M} \mathrm{NaClO}_{4} .50$ $\mathrm{mM}$ Tris, pH 8.5 at $23^{\circ} \mathrm{C}$.

\begin{tabular}{lccc}
\hline & $\begin{array}{c}K_{\text {obsd }} \\
(\mu \mathrm{M})\end{array}$ & $\begin{array}{c}k_{\text {on }} \\
\left(\mathrm{nM}^{-1} \mathrm{~s}^{-1}\right)\end{array}$ & $\begin{array}{c}k_{12} 2^{h} \\
\left(\mathrm{nM} \mathrm{s}^{-1}\right)\end{array}$ \\
\hline Control & $0.23 \pm 0.07$ & $0.53 \pm 0.03$ & $0.81 \pm 0.26$ \\
$+30 \mathrm{mM} \mathrm{Ca}\left(\mathrm{ClO}_{4}\right)_{2}$ & $0.50 \pm 0.05$ & $0.35 \pm 0.03$ & $1.19 \pm 0.16$ \\
\hline
\end{tabular}

"Evaluation of constants was by method A (see Table I) with the use of eq $14 \mathrm{ff}$. ${ }^{b} k_{12}$ was calculated from the equation $k_{12}=k_{\text {on }}$. $K_{\text {obsd }} / K_{1}$ assuming $K_{1}=0.149 \pm 0.003 \mu \mathrm{M}$ (Table I).

reasonable agreement with a value of $\sim 100 \mathrm{~s}^{-1}$ estimated from dilution experiments involving $N$-methylacridinium and acetylcholinesterase (Mooser and Sigman, 1975).

Effect of Inhibitors on Relaxation Times. The effect of $\mathrm{Ca}^{2+}$ on the relaxation times observed with acetylcholinesterase and $N$-methylacridinium was observed in Tris buffer at $\mathrm{pH}$ 8.5. As noted above, a slow decrease in ligand fluorescence was particularly pronounced in the absence of $\mathrm{Ca}^{2+}$. Ligand fluorescence decreased about $6 \%$ over the course of the relaxation experiments at each $c_{\mathrm{L}}{ }^{10 t}$ in the absence of $\mathrm{Ca}^{2+}$, while in the presence of $30 \mathrm{mM} \mathrm{Ca}^{2+}$ about $2 \%$ of the free ligand fluorescence was lost at each $c_{\mathrm{L}}{ }^{\text {tot }}$.

At the $1.0 \mu \mathrm{M} c^{2} E^{\text {init }}$ conditions in these studies, Schemes I and IV were adequate approximations and relaxation times were analyzed according to eq 14 ( method A). Data are given in Table II. Estimates of $k_{\text {on }}$ by method B were within experimental error of those listed in Table II. Both $\mathrm{Ca}^{2+}$ and Tris appear to act as competitive inhibitors. For $\mathrm{Ca}^{2+}, K_{1} \simeq 30$ $\mathrm{mM}$; for Tris at this $\mathrm{pH}, K_{\mathrm{I}} \simeq 50 \mathrm{mM}$.

Relaxation Measurements with Acetylcholinesterase and 1-Methyl-7-hydroxyquinolinium. Analysis of this interaction is slightly complicated by the fact that the ligand exists in two forms, the protonated cation and the unprotonated zwitterion. Only the zwitterion is fluorescent under the experimental conditions (Prince, 1966; Rosenberry and Bernhard, 1971). but our current study indicates that only the protonated form has significant affinity for the enzyme active site. The apparent ligand dissociation constants $K_{\text {obsd }}$ at three $\mathrm{pH}$ values are shown in Table III. The high $K_{\text {obsd }}$ value at $\mathrm{pH} 7.5$ suggests that the protonated ligand form has higher enzyme affinity. To analyze this suggestion quantitatively, Schemes IV and V were combined. Protons are known to act as competitive inhibitors of cation binding to the catalytic site of the enzyme with a $\mathrm{p} K_{1}$ of 6.3 (see Rosenberry, 1975b), and the ligand has a $\mathrm{p} K_{\mathrm{a}}$ of 5.9 (Prince, 1966; Rosenberry and Bernhard, 1971). In this case, $K_{\text {wbsd }}=K_{1}\left(1+\bar{c}_{H} / K_{1}\right)\left(1+K_{a} / \bar{c}_{H}\right)$. Calculated values of $K_{1}$ at each pH are also shown in Table III, and their agreement provides confirmation of this analysis.

Because the affinity of this ligand is greatly reduced at high $\mathrm{pH}$, it was necessary to study relaxation phenomena at a $\mathrm{pH}$ lower than 8.0. Only a single relaxation time was observed at $\mathrm{pH} 7.0$, and thus the study was carried out at this $\mathrm{pH}$ : at lower pHs, a complex relaxation spectrum was observed as noted above. At pH 7.0, $\tau_{\text {obsd }}{ }^{-1}$ followed eq $15(\operatorname{method} \mathrm{A})$. The observed value of $k_{\text {on }}$ was $0.162 \pm 0.011 \times 10^{9} \mathrm{M}^{-1} \mathrm{~s}^{-1}$. This corresponds to a calculated $k_{12}$ of $2.18 \pm 0.15 \times 10^{9} \mathrm{M}^{-1} \mathrm{~s}^{-1}$ (see eq $15 \mathrm{ff}$ ). The observed value of $k_{21}$ was $444 \pm 43 \mathrm{~s}^{-1}$. In the calculation of $k_{12}, k_{\text {on }}$ was assumed to be a function of $K_{\text {at }}$ but not of $K_{\mathrm{I}}$, in contrast to $K_{\text {obsd. }}$. This assumption was based on two observations: the relaxation time for ligand protonation was too small to be observed, consistent with the assumption above eq 15; and inhibition due to enzyme protonation is more complicated than the simple competition proposed in Scheme
TABLE III: pH Dependence of the Equilibrium Binding of

1-Methyl-7-hydroxyquinolinium to Acetylcholinesterase in $0.1 \mathrm{M}$ Sodium Phosphate.

\begin{tabular}{ccc}
$\mathrm{pH}$ & $\begin{array}{c}K_{\text {obsd }}{ }^{\mu} \\
(\mu \mathrm{M})\end{array}$ & $\begin{array}{c}K_{1}{ }^{b} \\
(\mu \mathrm{M})\end{array}$ \\
\hline 6.0 & $2.71 \pm 0.05$ & $0.40 \pm 0.01$ \\
7.5 & $18.6 \pm 1.7$ & $0.44 \pm 0.04$ \\
7.0 & $6.0 \pm 1.5$ & $0.37 \pm 0.09$ \\
\hline
\end{tabular}

" $K_{\text {obsd }}$ at pH 6.0 and 7.5 was determined at $25^{\circ} \mathrm{C}$ from a plot of $f c_{L^{t o t}} /\left(f c_{L^{2}}{ }^{10 t}-F\right)$ vs. $1 / c_{E^{t o t}}$ (eq 4 in Mooser et al., 1972). This plot has the additional advantage of confirming that enzyme-bound 1 methyl-7-hydroxyquinolinium is devoid of detectable fluorescence. $K_{\text {obsd }}$ at $\mathrm{pH} 7.0$ was obtained at $23^{\circ} \mathrm{C}$ by analysis according to eq 1 . ${ }^{n} K_{1}$ was determined from the equation $K_{1}=K_{\mathrm{obsd}} /\left(1+\bar{c}_{\mathrm{H}} / K_{\mathrm{I}}\right)(\mathrm{I}$ $+K_{\mathrm{a}} / \bar{c}_{\mathrm{H}}$ ), where $\mathrm{p} K_{\mathrm{l}}=6.30$ and $\mathrm{p} K_{\mathrm{a}}=5.90$ (see text). The indicated standard error reflects the error in $K_{\text {obsd }}$ only.

IV and apparently occurs slowly enough to give multiple coupled relaxations. Thus, any effect of enzyme protonation on $\tau_{\text {ubsd }}{ }^{-1}$ could not be analyzed by the given equations and was ignored. Such effects should be small at pH 7.0.

\section{Discussion}

Accuracy of Data. The equilibrium dissociation constant $K_{1}$ for the interaction of $N$-methylacridinium and acetylcholinesterase in Table $I$ is in agreement with that observed by Mooser et al. (1972) when a slight difference in solvent is taken into account. Two independent methods of analysis in Table I agree quite well on the value of $k_{12}$ which characterizes this interaction. The $k_{12}$ value is very high and is discussed further below. Method B is introduced in this report and is an extension of a procedure introduced by Eigen and Winkler-Oswatitsch (1977); it is particularly elegant in that only one experimental variable, the total ligand concentration $c_{L}{ }^{\text {tot }}$, need be known. From a single set of reiaxation times at constant total enzyme concentration, this method allows simuitaneous determinations of the bimolecular reaction rate constant, the equilibrium dissociation constant, and the toial enzyme normality for the liganc-enzyme interaction. Furthermore, these determinations are accomplished by a rigorous least-squares solution of a second-order polynomial which can include appropriate variance analyses.

The greatest uncertainty in our observed values arises from the estimate of $c_{\mathrm{L}}{ }^{\text {tot }}$ and is due to the progressive loss in fluorescence with time at each $c_{\mathrm{L}}{ }^{\text {tot }}$. Because this loss is increased at high $\mathrm{pH}$ and decreased at low $\mathrm{pH}$ and in the presence of $\mathrm{Ca}^{2+}$, it quite plausibly could arise from adsorption of the positively charged ligand on the quartz walls of the temperature-jump cell. An alternative explanation for the loss is suggested by a recent report on the photochemical dismutation of $N$-methylacridinium (Einarsson and Zeppezauer, 1975), a process catalyzed by either horse liver alcohol dehydrogenase or human serum albumin. A similar photochemical sensitivity is much less apparent in our experiments. Addition of acetylcholinesterase, if anything, decreases the rate of fluorescence loss, and the rate of loss is not greatly affected by whether the exposure to excitation light is intermittant or continuous.

The effect of uncertainty in $c_{L}{ }^{\text {tot }}$ arising from this source on the kinetic rate constants is not large. If $c^{c}{ }^{\text {tot }}$ is not corrected for fluorescence loss in method B in Figure 5, for example, $k_{12}$ is reduced by $11 \%, K_{1}$ is increased by $19 \%$, and $c_{\mathrm{E}}{ }^{\text {init }}$ is increased by $8 \%$. The uncertainty in $\mathrm{cL}_{\mathrm{L}}{ }^{\mathrm{t} t}$ also affects the estimate of the fluorescence coefficient $f$. Figures $2-5$ were plotted in the original units of photomultiplier voltage, but to convert to $\mu \mathrm{M}$ units in Figures $2-5$ and Tables I- III a division by $f$ was 
carried out. In this conversion $f$ was considered to have insignificant error, and thus the indicated standard errors of the thermodynamic and kinetic constants reflect the precision of the data. Because the uncertainty in $c_{L}{ }^{\text {tot }}$ may have resulted in a standard error in $f$ of $5 \%$, the absolute standard errors are somewhat larger than those indicated.

Estimates of $k_{\text {on }}$ and $K_{\text {obsd }}$ obtained by applying method B to a variety of relaxation data indicate a close correlation of $k_{\text {on }}$ and $k_{21} \equiv k_{\text {on }} K_{\text {obsd }}$ with the corresponding values of $k_{\text {on }}$ and $k_{21}$ obtained from method A (eq 14). In other words, when the application of method $\mathrm{A}$ alone indicates a discrepancy between the equilibrium determination of $K_{\text {obsd }}$ (eq 1 ) and the kinetic determination of $K_{\text {obsd }}=k_{21} / k_{\text {on }}$ (eq 14) (e.g., the data on compound II above), the method B estimate of $K_{\text {obsd }}$ agrees with the kinetic estimate of method A. Such discrepancies probably indicate systematic inconstancies in the data, as noted above. With the appropriate computer program, method B is very straightforward; because $\tau^{-2}$ values are used, appropriate weighting is essential. The agreement of estimates of $c_{\mathrm{E}}{ }^{\text {init }}$ and/or $K_{\text {obsd }}$ from this method with independent estimates from equilibrium data is an indication of the overall consistency of the data. The accuracy of method B appears greatest when $K_{\text {obsd }} \leq c_{E}{ }^{\text {init }} \leq 5 K_{\text {obsd }}$ under which conditions the plot corresponding to Figure 5 has an inverted bell shape.

Mechanistic Schemes Examined. Over most of the concentration ranges examined, the interactions of both $N$ methylacridinium and 1-methyl-7-hydroxyquinolinium with acetylcholinesterase appear consistent with Scheme I. Only at high enzyme concentrations does $N$-methylacridinium begin to show a second, faster relaxation time. While the data indicate that this second relaxation is best explained by Scheme II', appropriate coupling of the two equilibria in Scheme III could have given qualitatively similar observations. A plausible candidate for the second ligand-binding site postulated by Scheme II' is the peripheral anionic site on acetylcholinesterase defined by the binding of propidium (Taylor and Lappi, 1975). This peripheral site is probably the anionic site approximately $14 \AA$ away from the catalytic site between which bisquaternary ligands specifically bind to acetylcholinesterase (Mooser et al., 1972; Taylor and Lappi, 1975; Wee et al., 1976; see Rosenberry, 1975a). While no relaxation spectrum consistent with Scheme III was observed, it is likely that that binding of $N$ methylacridinium to the catalytic site of acetylcholinesterase does induce a conformational change of the enzyme-ligand complex. Aromatic cation binding has been suggested to involve conformational changes of acetylcholinesterase (Rosenberry and Bernhard, 1972; Rosenberry, 1975a), and $N$ methylacridinium is one of only a few aromatic cations which can accelerate the acetylcholinesterase-catalyzed hydrolyses of methyl or ethyl acetate (Barnett and Rosenberry, 1977). The acceleration phenomenon is highly suggestive of an "induced-fit" ligand-enzyme complex (Koshland, 1958; Rosenberry, 1975b). Induced fit can be formalized by Scheme III, but if this process occurs with $N$-methylacridinium and acetylcholinesterase the rate constants for the second step are sufficiently fast that this step rapidly equilibrates and, hence, is not observable.

Basis of High $k_{12}$ Values. The most important finding in our study is the high values of $k_{12}$ that characterize the binding of both $N$-methylacridinium and 1-methyl-7-hydroxyquinolinium to the catalytic site of acetylcholinesterase. These $k_{12}$ values are greater than $10^{9} \mathrm{M}^{-1} \mathrm{~s}^{-1}$. Typical values of $k_{12}$ for the interaction of ligands with specific enzyme sites are in the range of $10^{7}$ to $10^{8} \mathrm{M}^{-1} \mathrm{~s}^{-1}$, although $k_{12}$ values for NADH and certain dehydrogenases approach $10^{9} \mathrm{M}^{-1} \mathrm{~s}^{-1}$ (see Hammes and Schimmel, 1971). An example of ligand binding closely analogous to those reported here is the interaction of the aromatic cation proflavin with the active site of chymotrypsin. A $k_{12}$ of only $1 \times 10^{8} \mathrm{M}^{-1} \mathrm{~s}^{-1}$ at $12{ }^{\circ} \mathrm{C}$ and $\mathrm{pH} 9.2$ was observed (Havsteen, 1967).

It is instructive to consider the theoretical encounter frequency $k_{12}$ of a ligand with an enzyme active site in aqueous solution. A first approximation of $k_{12} *$ can be made on the basis of eq 18 (see, e.g., Eigen, 1974; Eigen and Hammes, 1963) for the association step of the cationic ligand (charge number $z_{L}=+1$ ) with the active site of the enzyme which carries at least one negative charge $\left(z_{\mathrm{E}}=-1\right)$.

$$
k_{12} *=\frac{2 \pi N}{10^{3}}\left(D_{\mathrm{E}}+D_{\mathrm{L}}\right)\left(d_{\mathrm{EL}}\right)(\alpha)
$$

In eq $18, N$ is Avogadro's number, $2 \pi$ is the physically plausible value for the solid angle of diffusional approach of the ligand to the active site, $D_{\mathrm{E}}+D_{\mathrm{L}} \simeq D_{\mathrm{L}} \simeq 10^{-5} \mathrm{~cm}^{2} / \mathrm{s}$ is the estimate for the sum of the diffusion coefficients of enzyme and ligand, respectively, and $d_{\mathrm{EL}} \simeq 5 \AA$ is the estimated "encounter distance" within which $\mathrm{E}$ and $\mathrm{L}$ react to form a complex. The term $\alpha$ accounts for the electrostatic contribution to the association. To a first approximation for an ionic strength of $\sim 0.1$ $\mathrm{M}$ and with $z_{\mathrm{L}} z_{\mathrm{E}}=-1$, we may use $\alpha \simeq 1$. From eq 18 we then estimate $k_{12}{ }^{*} \simeq 2 \times 10^{9} \mathrm{M}^{-1} \mathrm{~s}^{-1}$. Since the observed $k_{12}$ values in this study approach this $k_{12} *$ estimate, the bimolecular reactions studied here approach diffusion control as defined by Eigen and Hammes (1963); i.e., any encounter between ligand and active site leads to a complex, independent of approaching ligand orientation. If the effective charge number $z_{\mathrm{E}}$ of the active site is increased by neighboring fixed (negatively) charged groups, a larger electrostatic contribution results in a larger value of $\alpha$ and thus a larger value of $k_{12} *$. Even in this event, the observed $k_{12}$ values are close to diffusion controlled.

It is of interest to consider whether any other mechanisms underlie the extremely high association rates with which acetylcholinesterase is able to react with cationic ligands like $N$-methylacridinium or 1-methyl-7-hydroxyquinolinium ions. Recently; Eigen (1974) discussed a way in which macromolecules can achieve $k_{12}$ values for specific ligand interactions that exceed the apparent theoretical maximum of $k_{12}$. If such macromolecules form extended surfaces in one (DNA) or two dimensions (membrane-bound proteins) and have numerous nonspecific binding sites, ligand encounters at nonspecific sites followed by rapid surface diffusion to the specific binding site can provide a considerable increase in the effective $d_{E L}$ in eq 18. Such an increase, amounting to two or three orders of magnitude, appears to obtain for the interaction of lac repressor with the operator site on DNA.

Acetylcholinesterase possesses several peripheral anionic sites for which, as yet, no physiological function has been found (see Rosenberry, 1975a). Since the $k_{12}$ values with aromatic cations here as well as the apparent bimolecular reaction rates obtained from steady-state studies with acetylcholine and other specific substrates (Rosenberry, 1975a; Rosenberry, 1975b) are somewhat higher than normally observed from enzymeligand interactions, it is pertinent to inquire whether surface diffusion enhances these rates. One indication of a surfacediffusion mechanism which is based on electrostatic interactions between ionic ligands and fixed surface charges is a high ionic strength dependence of $k_{12}$ (see Eigen, 1974). Increasing ionic strength tends to increase the dissociation rate constant from nonspecific sites and hence to reduce the surface diffusion contribution to $d_{\mathrm{EL}}$. While in principle it would thus be relevant to investigate the ionic-strength dependence of ligand interactions with acetylcholinesterase, the enzyme undergoes rather 
striking changes in ligand-binding properties as the ionic strength is raised from 0.001 to $0.1 \mathrm{M}$ (Mooser and Sigman, 1974; Taylor and Lappi, 1975) which suggest multiple enzyme conformations. To avoid complications in analysis which could arise from ionic-strength-dependent enzyme conformational equilibria, we investigated the role of peripheral anionic sites by introducing $30 \mathrm{mM} \mathrm{Ca}\left(\mathrm{ClO}_{4}\right)_{2}$ to the control solvent, 0.1 $\mathrm{M} \mathrm{NaClO} 4,50 \mathrm{mM}$ Tris, $\mathrm{pH} 8.5 . \mathrm{Ca}^{2+}$ is known to bind with high affinity to peripheral anionic sites and much lower affinity to the catalytic site of acetylcholinesterase (Roufogalis and Quist, 1972; Taylor and Lappi, 1975). If peripheral anionic sites contribute to a surface diffusion component in the $k_{12}$ values observed here, this introduction of $\mathrm{Ca}^{2+}$ should significantly reduce these values. No such contribution was found; $30 \mathrm{mM} \mathrm{Ca}^{2+}$ reduced $k_{\text {on }}$ by less than a factor of 2 (Table II) or to about the extent expected for competition at the catalytic site (Scheme IV) with a $K_{\mathrm{I}}$ for $\mathrm{Ca}^{2+}$ of about $30 \mathrm{mM}$. The apparent absence of a surface diffusion contribution from peripheral sites to $k_{12}$ at the roughly physiological ionic strength used here does not rule out the possibility of such a contribution from other surface sites with $\mathrm{Ca}^{2+}$ dissociation constants $>30 \mathrm{mM}$.

Physiological Relevance of High $k_{12}$ Values. Acetylcholine in physiological systems is largely compartmentalized intracellularly such that it remains protected from hydrolysis by acetylcholinesterase located on the external cell surface (see Rosenberry, 1975a). During excitation, acetylcholine is rekased, and acetylcholinesterase and acetylcholine receptors, present in about equal amounts in excitable membranes (see Rosenberry, 1975a), compete for the released acetylcholine. The high value of $2 \times 10^{8} \mathrm{M}^{-1} \mathrm{~s}^{-1}$ for $k_{\text {eat }} / K_{\text {app }}$ for acetylcholine noted in the Introduction defines a minimum value for the bimolecular rate constant for the reaction of acetylcholine with acetylcholinesterase. The even higher values of $k_{12} \mathrm{ob}-$ served for the fluorescent ligands in this report suggest that this constant may even be higher. In any event, the destruction of acetylcholine by acetylcholinesterase is close to diffusion controlled. In contrast, a recent temperature-jump relaxation kinetic study found that acetylcholine interacts with solubilized and purified acetylcholine receptor with a $k_{12}$ of $2 \times 10^{7} \mathrm{M}^{-1}$ $s^{-1}$ (Neumann and Chang, 1976), an order of magnitude lower than the second-order enzyme-catalyzed hydrolysis rate. Thus, the competition of these two proteins in solution for acetylcholine would greatly favor acetylcholinesterase. Since the interaction of acetylcholine with receptor is vital to consequent membrane conductance changes, the in vivo structural organization inherent in the membrane localization of the two proteins may prevent them from competing for acetylcholine as they would in homogeneous solution.

\section{Acknowledgments}

We thank Professor M. Eigen for helpful discussions of this work. IMSL computer subroutine programs were brought to our attention by Jim Odell, who helped adapt them to our use in this study.
References

Barnett, P., and Rosenberry, T. L. (1977), J. Biol. Chem. (in press).

Eigen, M. (1974), in Quantum Statistical Mechanisms in the Natural Sciences, Mintz, S. L., and Widmayer, S. M., Ed., New York, N.Y., Plenum Press, pp 37-61.

Eigen, M., and DeMaeyer, L. (1963), Tech. Org. Chem. 8, Part 2, 895-1054.

Eigen, M., and Hammes, G. G. (1963), Adv. Enzymol. 25, $1-38$.

Eigen, M., and Winkler-Oswatitsch (1977), Angew. Chem. (in press).

Einarsson, P., and Zeppezauer, M. (1975), Eur. J. Biochem. $59,295-304$.

Forsythe, G. E. (1957), J. Soc. Indust. Appl. Math. 5, 7488.

Hammes, G. G, and Schimmel, P. R. (1971), Enzymes, 3rd Ed. 2, 109.

Havsteen, B. H. (1967), J. Biol. Chem. 242, 769-771.

Kelly, L. G. (1967), Handbook of Numerical Methods and Applications, Reading, Mass., Addison-Wesley, p 68.

Koshland, D. E., Jr. (1958), Proc. Natl. Acad. Sci. U.S.A.44, 98.

Kremzner, L. T., and Wilson, I. B. (1964), Biochemistry 3, 1903.

Lawler, H. C. (1961), J. Biol. Chem. 236, 2296.

Michel, H. O., and Krop, S. (1951), J. Biol. Chem. 190, 119.

Mooser, G., and Sigman, D. S. (1974), Biochemistry 13. 2299-2307.

Mooser, G., and Sigman, D. S. (1975), Croat. Chem. Acta 47. 265-275.

Mooser, G., Schulman, H., and Sigman, D. S. (1972), Biochemistry ll, 1595-1602.

Neumann, E., and Chang, H. W. (1976), Proc. Natl. Acad. Sci. U.S.A. 73, 3994-3998.

Prince, A. K. (1966), Biochem. Pharmacol. 15, 411-417.

Riegler, R., Rabl. C.-R., and Jovin, T. M. (1974), Ret. Sei. Instrum. 45, 580-588.

Rosenberry, T. L. (1975a), Adv. Enzymol. 43, 103-218.

Rosenberry, T. L. (1975b), Proc. Natl. Acad. Sci. U.S.A. 72. 3834-3838.

Rosenberry, T. L., and Bernhard. S. A. (1971), Biochemistry 10, $4114-4120$

Rosenberry, T. L., and Bernhard, S. A. (1972), Biochemistry 11,4308-4321.

Rosenberry, T. L., Chen, Y. T., and Bock, E. (1974), Biochemistry 13, 3068-3079.

Roufogalis, B. D., and Quist, E. E. (1972), Mol. Pharmacol. $8,41$.

Taylor, P., and Lappi, S. (1975), Biochemistry 14, 1989. 1997.

Thusius, D. (1972), J. Am. Chem. Soc. 94, 356-363.

Wee, V. T., Sinha, B. K., Taylor, P. W., and Chignell. C. F. (1976), Mol. Pharmacol. 12, 667-677. 